\title{
PDTA e Medicina di Laboratorio
}

\section{Care pathways and Laboratory Medicine}

\author{
Piero Cappelletti ${ }^{1}$
}

Ricevuto: 30 maggio 2017 / Accettato: 12 giugno 2017 / Pubblicato online: 30 giugno 2017

(C) Società Italiana di Patologia Clinica e Medicina di Laboratorio 2017

Riassunto I Percorsi Diagnostico-Terapeutici Assistenziali (PDTA) sono definiti come interventi complessi che descrivono i processi delle decisioni cliniche e dell'organizzazione di cura di un ben definito gruppo di pazienti, in un definito periodo di tempo. Costituiscono i pilastri per la realizzazione della "centralità del paziente" sulla base delle evidenze scientifiche. I PDTA possono essere definiti come la contestualizzazione di una o più linee guida, relative a una patologia o problematica clinica, nella specifica realtà organizzativa di un'Azienda sanitaria. I PDTA sono ampiamente utilizzati ed esistono evidenze, seppur narrative, della loro efficacia. I principali problemi sono la personalizzazione e l'innovazione. La Medicina di Laboratorio deve fare la sua parte nella costruzione dei PDTA, partecipando ai gruppi multidisciplinari e multiprofessionali, con attenzione ai test pivot, al loro uso e alla loro interpretazione; alla qualità delle linee guida scelte; al dettaglio dei protocolli operativi che sostanziano il percorso; agli aspetti "interni" che determinano (performance analitica) o aumentano (total testing process) la qualità della risposta; alla verifica continua dell'appropriatezza e adeguatezza dei marcatori utilizzati e all'audit dell'effectiveness del PDTA. Si tratta di uno degli aspetti più alti di "lavoro all'interfaccia", nelle aree di pre-pre e post-post testing process, ove l'affidabilità analitica e il suo rapporto con la realtà clinica e i suoi esiti sono immediatamente percepibili.

Parole chiave Medicina di Laboratorio - Percorsi Diagnostico-Terapeutici Assistenziali (PDTA) · PIC · Centralità del paziente $\cdot$ Linee guida $\cdot$ Performance analitica

$\triangle$ P. Cappelletti pie.cappelletti@gmail.com

1 SIPMeL, Castelfranco Veneto, TV, Italia
Summary A care pathway $(C P)$ is a complex intervention for the mutual decision making and organization of care processes for a well-defined group of patients during a welldefined period. The aim of a CP is to enhance the quality of care across the continuum by improving risk-adjusted patient outcomes, promoting patient safety, increasing patient satisfaction, and optimizing the use of resources. The structured, multidisciplinary plan that details essential steps to improve continuity and coordination in the care of specific patients must be evidence based: $C P$ is a local translation and/or adaption of guidelines. The CPs are used worldwide and some evidences exist of its effectiveness. Main issues are the personalization and the innovation of the CPS. Laboratory Medicine has a crucial role in the multidisciplinary and multiprofessional team that builds the CP, for the appropriate use and interpretation of the pivot tests, choosing and adapting guidelines, defining protocols using laboratory tests, delivering high quality results and interpretations, verifying effectiveness of the processes, and auditing the outcomes. It is a high value activity of work at the laboratoryclinical interface, where the direct or indirect relationship between analytical performance, total quality and clinical outcomes is immediately visible.

Keywords Laboratory Medicine - Care pathways · Clinical pathways · Patient-centered medicine - Guidelines . Analytical performance

\section{Centralità del paziente e PDTA}

La "centralità del paziente" [1] è divenuta un tema cruciale della Medicina occidentale, anche per la presa d'atto delle iniquità, frammentazioni, sovrapposizioni e discontinuità che caratterizzano molto spesso l'erogazione dei servizi sanitari (effetto silo) e delle loro conseguenze sugli esiti 
clinici, psicosociali e sullo spreco di risorse umane ed economiche. Essa è di particolare importanza per la gestione delle malattie croniche, che oggi rappresentano il grande problema sanitario del mondo [2].

Nel 1996 Wagner et al [3] identificarono 5 elementi necessari per migliorare gli esiti dei pazienti cronici (Evidence-based planned care; Reorganization of practices to meet the needs of patients who require more time, a broad array of resources, and closer follow-up; Systematic attention to patients' need for information and behavioral change; Ready access to necessary clinical expertise; Supportive information systems) e nel 1998 proposero un innovativo modello di sanità integrata (Chronic Care Model, CCM) [4], che si basa sulla fruttuosa interazione tra un paziente informato e attivo e un team sanitario preparato e proattivo. Gli elementi essenziali sono: il self-management come parte della collaborazione del paziente; una medicina proattiva, integrata e di prossimità; supporti di evidenze in particolare linee guida (LG); un sistema informativo clinico sempre accessibile e tempestivo. Il suo adattamento internazionale è il WHO Innovative Care for Chronic Conditions (ICCC) Framework [5], nel quale la diade paziente-team diviene una triade "paziente-team-comunità" [2]. Esistono evidenze narrative di effetti positivi di tale approccio, quali una maggior soddisfazione dei pazienti e del personale, migliori esiti primari (mortalità) e secondari (dimissioni e riammissioni, lunghezza della degenza), diminuzione degli errori e percezione dello staff di un cambiamento positivo nell'assistenza [6].

Il corrispettivo anglosassone di CCM è l'Integrated $\mathrm{Ca}$ $r e[7,8]$, le cui differenze sono: parte dalla realtà ospedaliera per andare verso il territorio; affronta temi più specifici; ha come punto centrale la continuità delle cure piuttosto che il rapporto qualità/costo. Entrambe hanno come punti salienti la coordinazione degli interventi e la centralità del paziente. I pazienti complessi, in modo particolare, richiedono misure di Integrated Care: coordinazione attraverso specialisti, strutture e supporti (per esempio, simultaneous care per la palliazione); mantenute nel tempo e tra gli episodi; tagliata sui bisogni e preferenze del paziente e basata sulla responsabilità condivisa di pazienti e caregivers.

I pilastri per la messa in pratica dell'integrated care e/o del CCM sono gli integrated care pathways (anglosassoni) o i clinical pathways (americani), conosciuti in Italia come PDTA (Percorsi Diagnostico-Terapeutici Assistenziali).

\section{Percorsi Diagnostico-Terapeutici Assistenziali (PDTA)}

I PDTA sono nati a metà degli anni Ottanta a Boston, nel New England Medical Center, e da lì si sono diffusi a ondate successive prima in Nord America (in Canada come CareMaps), poi nel mondo anglosassone e del Commonwealth, quindi in Europa e Giappone e infine dopo il 2000 anche in aree sudamericane e del sud-est asiatico [8]. Tuttavia, come ricorda il sito di EPA (European Pathways Association) [9], ancora oggi vi sono molteplicità di termini e incertezze di contenuto.

Nel 2001 De Luc [10] ha annotato 17 sostantivi diversi (Care pathways, Critical pathways, Care Maps, Anticipated recovery plans, Collaborative care plans, Care profiles, $\mathrm{Ca}$ re protocols, Clinical progression, Clinical outcomes ecc.) che descrivono questo concetto in letteratura e, nel 2006, de Bleser et al [11] hanno raccolto 84 diverse definizioni di Clinical Pathway (CP) in 82 dei 263 articoli selezionati con la metodologia della "concept analysis". Gli Autori sostengono che nessuno dei 19 termini ulteriormente selezionati contiene totalmente le 2 dimensioni considerate critiche (contenuto e cambiamento), obiettivo che è avvicinato da "linee guida" ma probabilmente potrebbe essere meglio espresso da "processo" o "intervento". Campbell et al [7] stressano il rapporto con le LG quali sintesi delle "evidenze", nei parametri identificativi dei CP. Vanaecht et al [12], attraverso un audit, intendono verificare se i criteri accettati (multidisciplinarietà, base di evidenze, gestione della variabilità clinica) consentono di individuare correttamente i CP analizzati. Kinsman et al [13] stilano 5 criteri (multidisciplinarietà, traslazione locale di LG, percorso dettagliato in passaggi spazio-temporali, criteri della progressione temporale, standardizzazione di un percorso di cura) per definire un $\mathrm{CP}$.

La terminologia confusa è causa ed effetto di una confusa comprensione. Tuttavia, la definizione oggi largamente accettata è quella fornita da EPA [9]. PDTA è un complesso intervento che definisce i processi delle decisioni cliniche $e$ dell'organizzazione di cura di un ben definito gruppo di pazienti, in un definito periodo di tempo. Le caratteristiche comprendono: la definizione di obiettivi ed elementi chiave di cura basati sull'evidenza (in particolare LG), la best practice e le aspettative dei pazienti; la facilitazione della comunicazione tra professionisti e con il paziente e familiari; la coordinazione spazio-temporale dei processi di cura e delle attività del team multidisciplinare e multiprofessionale, del paziente e familiari; documentazione, monitoraggio e valutazione della variabilità e outcome; e l'identificazione di appropriate risorse. L'obiettivo è il miglioramento della qualità di cura lungo il continuum dell'assistenza, migliorando gli esiti aggiustati per il rischio, promuovendo la sicurezza del paziente e ottimizzando le risorse.

In Italia, si tende a enfatizzare l'ambito di estensione del PDTA, qualificandolo come ospedaliero e/o territoriale. Quando un PDTA descrive il processo, concernente uno specifico problema di salute, nella sua gestione sia territoriale sia ospedaliera si parla di Profilo Integrato di Cura (PIC) [14].

Nella pianificazione e sviluppo di un PDTA vi sono dei punti salienti, correttamente indicati da Campbell et al [7] 
ancora nel 1998 (Select an important area of practice; Gather support for the project; Form a multidisciplinary group; Identify established guidelines; Review practice; Involve local staff; Identify key areas for service development; Develop an integrated care pathway; Prepare documentation; Educate staff; Pilot implementation; Regularly analyse variables; Discuss variations). In ambito italiano, GIMBE [14] ha definito un percorso più snello in 5 fasi (Definizione priorità; Costituzione del gruppo di lavoro; Mappatura del percorso; Elaborazione e stesura del Percorso Assistenziale; Disseminazione, Implementazione, Valutazione).

In primo luogo si devono definire le priorità come area d'interesse (esiti desiderabili e criteri di eleggibilità) e come gruppo omogeneo di pazienti. L'area d'interesse è di solito focalizzata a malattie ad alto costo e alta prevalenza e dipende dalla disponibilità di terapie multiple e di pattern di trattamento eterogenei. Prospetticamente l'oncologia è l'area a più elevata probabilità di espansione $(85 \%)$ di PDTA, seguita da altre aree come la reumatologia, la cardiologia, il diabete con il $65 \%$. Nelle aree terapeutiche le motivazioni sono meglio stabilite: costo $(62 \%)$, variabilità della pratica (57\%), esiti (33\%) e case mix (19\%) [15]. La scelta di un gruppo omogeneo ben definito di pazienti è sostanzialmente soggettiva: de Bleser et al [11] citano almeno 12 criteri diversificati.

Un secondo punto critico è la formazione del gruppo di lavoro. Nel formare il gruppo che deve, per definizione, essere multidisciplinare bisogna tenere conto anche della multi-professionalità (infermieri soprattutto), ma non dimenticare che i clinici possono essere la più forte barriera all'espansione dei PDTA [15].

Il punto centrale è l'uso delle evidenze sotto forma di LG. Il PDTA, infatti, può essere definito come la contestualizzazione di una o più $L G$, relative a una patologia o problematica clinica, nella specifica realtà organizzativa di un'Azienda sanitaria. In una recente ricerca americana [15], LG (86\%) e RCT (81\%) sono le fonti "chiave" per lo sviluppo di PDTA, più ancora delle metanalisi $(63 \%)$. Inoltre, 1 ' $81 \%$, il $71 \% \mathrm{e}$ il $57 \%$ dei rispondenti, rispettivamente, indicano il costo dei farmaci, i costi totali e l'uso di risorse come elementi importanti. Altre fonti che interessano circa la metà dei rispondenti, nonostante l'indubbio valore, sono la possibile personalizzazione del PDTA (48\%) e la comparazione indiretta di interventi diversificati (43\%). Di solito, infine, i processi e la pratica dell'implementazione non sono menzionati. Infatti, non vi è uno standard riconosciuto per l'implementazione delle LG nei PCTA. L'aderenza alle LG è oggi considerata la misura dell'appropriatezza, nell'accezione di rapporto con quesito clinico e outcome. Le LG e le loro caratteristiche necessarie (standard) sono state definite anche recentemente da IOM e per la loro valutazione si concorda largamente sull'utilizzo del metodo AGREE II [14]. Ma è proprio l'implementabilità delle LG a essere spesso un problema. In un lavoro dedicato a questo tema, Gagliardi et al [16] hanno esaminato venti LG per malattie croniche, segnalando come le condizioni cliniche di riferimento siano esplicitate nel 90\% e un evidence grading system nel $95 \%$ dei casi. Gli strumenti applicativi delle indicazioni di LG come algoritmi e sintesi delle raccomandazioni sono presenti nel 65\% e 55\% dei casi, rispettivamente. Tuttavia, solo il 50\% fornisce informazioni per il paziente, meno del $50 \%$ informazioni tecniche (45\%), normative (15\%), di risorse umane (5\%), o di competenza professionale (20\%) per l'implementazione. Il $45 \%$ afferma che devono essere promosse ma non dice come; solo il $30 \%$ fornisce elementi per la valutazione in itinere (checklist) e il 15\% identifica le possibili barriere; nessuna offre attenzione alle necessità degli stakeholders e ai costi. Le LG, infine, sono spesso troppo corpose con un valore di mediana per numero di pagine di 72,5 (da 21 a 878) e un valore di mediana per numero di raccomandazioni di 41,5 (da 8 a 214).

In conclusione, vi è grande attenzione alla clinica, alle evidenze e al loro grading, parziale al sommario delle raccomandazioni e all'educazione dei pazienti, poca per le informazioni necessarie all'implementazione e alla misura delle performance, molto poca all' assessment, alle necessità degli stakeholders, al costo del loro uso anche in termini di risorse umane e al dettaglio del processo d'implementazione. In altre parole, la gran parte delle LG non fornisce elementi per l'appropriatezza organizzativa inerente la loro implementazione. Si sono, comunque, valutati i Fattori Critici di Successo nell'implementazione dei PDTA [17, 18], seppure con esiti contraddittori. Tuttavia, si possono indicare, nell'ordine: consapevolezza del top management, consapevolezza e iniziativa bottom-up dei professionisti, sistemi di accreditamento, feedback (miglioramento continuo), capacità manageriali, sistema informativo, comunicazione, aderenza al PDTA (mantenimento), consapevolezza dei pazienti. Quanto alle barriere $[15,19]$, la principale è il non coinvolgimento dei clinici interessati (85\%); altre sono una comunicazione diretta inadeguata, un insufficiente sistema di tracciamento e documentazione, una mancata partecipazione del paziente, un eccessivo carico amministrativo, esiti e/o risparmi non dimostrabili.

Sulla base delle LG scelte, il gruppo costruisce il PDTA, lo valida, lo testa e lo implementa con mezzi educativi e il controllo di un network. I principali criteri per i PDTA di trattamento sono l'efficacia terapeutica, la tollerabilità e il costo, per un gruppo selezionato di pazienti e con il focus sul rapporto costo/efficacia [15]. Accanto a questi sono importanti i criteri di accesso dei pazienti, i percorsi diagnostici e le diramazioni dei percorsi secondo algoritmi. Lo strumento migliore per descrivere e rappresentare un PDTA/PIC è il diagramma di flusso, perché permette una visione globale dell'intero percorso e l'identificazione di episodi (attività o insieme di attività) maggiormente significativi che 
lo compongono. Talora è possibile tradurre le LG in metamodelli [20] e in strumenti informatici locali o commerciabili: negli USA ci sono diversi software già pronti, adattabili alla realtà locale, soprattutto di PDTA oncologici [21], talora preparati dalle Assicurazioni (e qui sorge un problema di trasparenza e di conflitto di interessi, se si pagano solo le prestazioni "on pathway").

Gli indicatori di qualità sono fondamentali per dimostrare in modo sintetico il "valore" del PDTA (esiti/costo), monitorare e raffinare la catena struttura-processo-outcome del PDTA durante il funzionamento in un confronto spaziotemporale contro standard o precedenti esperienze, dimostrare la qualità e convenienza nei sistemi "pay-forperformance" [22]. Possono individuare [14] indicatori di struttura, che misurano le risorse utilizzabili nell' ambito del percorso (operatori, dotazioni tecnologiche, posti letto ecc.); indicatori di processo, che misurano il funzionamento del PDTA/PIC; indicatori di appropriatezza clinica (diagnostica/terapeutica) oppure organizzativa (al domicilio, in ambulatorio, nel day-hospital, durante il ricovero); indicatori di output che misurano i volumi di produzione (giornate di degenza, prestazioni erogate) o la capacità produttiva come rapporto tra una dotazione strutturale (posti letto, numero personale) e il volume di produzione; indicatori di esito, che misurano le variazioni dello stato di salute a livello del singolo individuo e della collettività; indicatori di equilibrio economico, che misurano l'impatto economico del PDTA/PIC globale, come costo delle risorse impiegate nell'erogazione del percorso, e parziale, come costo di singole fasi del PDTA/PIC (parte ospedaliera, territoriale, ambulatoriale).

La verifica dell'applicazione dei PDTA è punto fondamentale. Può essere effettuata con diversi approcci che, comunque, prevedono una specifica modalità sistematica $\mathrm{e}$ condotta secondo uno specifico modello di lavoro, quale quello dell'audit sia esso clinico o organizzativo. Nella ricerca USA già citata [15], il 95\% cita come criterio principale il tasso di compliance, $\approx 70 \%$ metriche di qualità clinica, $\approx 50 \%$ il mantenimento dei pazienti nel PDTA e poco meno del $50 \%$ il risparmio di risorse ospedaliere (ricoveri, lunghezza della degenza). Tuttavia, poiché esiti, qualità, accesso e sostenibilità non sono facilmente misurabili, non vengono menzionati.

I PDTA sono ampiamente utilizzati: nel 2003 1'80\% degli ospedali americani li utilizzava [13]; nel sito di NICE [23] ne sono catalogati 454. In Italia [24] nel 2014, i PDTA attivi erano 338 (293 pienamente funzionanti): 129 in Lombardia, 67 in Emilia Romagna, 40 in Toscana e 26 nel Lazio; nel Sud sono complessivamente 44 , rilevati però solo in 8 aziende campione e quindi indice di una diffusione già soddisfacente. Le aree cliniche maggiormente coinvolte sono oncologia (n. $65 ; 20 \%$; in particolare per cancro mammario e polmonare), cardiologia (47), neurologia (35), endocrinologia (31), malattie respiratorie (24), ortopedia (18) e malattie rare (16).
Sono anche efficaci? Secondo la ricerca citata [24], i risultati in termini clinici ed economici mostrano una riduzione del $18 \%$ dei giorni di ricovero per le infezioni e del $20 \%$ per le polmoniti, mentre è del $42 \%$ la riduzione delle complicanze prevenibili in ospedale, grazie al miglior trattamento di infezioni, polmoniti ed emorragie. Anche in campo internazionale, sull'efficacia generale dei PDTA, esistono revisioni $[25,26]$ non sistematiche che suggeriscono impatti positivi sul costo (nel 2014 piuttosto che nel 2007) e sulla qualità delle cure (coordinazione, lunghezza della degenza, follow-up), più limitatamente su complicanze e riammissioni e ancora meno su mortalità e morbilità. Una metanalisi australiana [27] del 2012 evidenzia effetti positivi sulla diminuzione delle complicanze intraospedaliere e un importante miglioramento nella documentazione sanitaria. Tuttavia, mancano valutazioni adeguate dell'entità assoluta degli scostamenti positivi. In alcuni campi specifici si è cercato di misurare adeguatamente l'effectiveness dei PDTA. Per i PDTA oncologici la Value in Cancer Care Task Force di ASCO [28] ha definito una valutazione formalizzata, sulla base di tre effetti critici-beneficio clinico (efficacy), tossicità (sicurezza) e costo (efficienza/economicità)—che sono i più facilmente valutabili. I primi due sono combinati per generare il beneficio netto di salute (net health benefit, $N H B$ score) confrontato poi con il costo per dare una valutazione globale costo/beneficio (overall summary assessment, OS). Le metriche utilizzate sono QALY (Quality-Adjusted LifeYears) e ICER (Incremental Cost-Effectiveness Ratios). Ma la centralità del paziente, la tempestività della terapia e l'equità nell'accesso alle cure, che pure sono elementi essenziali di qualità per il paziente ma difficilmente misurabili, sono raramente riportati come outcome negli studi clinici.

I principali problemi dei PDTA sono due. Il primo è la violazione del PDTA quando si deve farlo per il bene del paziente: la compliance massima dei PDTA/PIC oncologici è a $80 \%$, a causa dell'eterogeneità dei tumori e dei pazienti. Questa consapevolezza è presente fin dai primi lavori [7] sull' integrated care, ma è chiaro che la difficoltà è l'equilibrata propensione del medico al mantenimento o alla fuoriuscita del singolo paziente nel/dal PDTA. Per superare la soggettività, vi sono proposte canadesi [29] di adattamento personalizzato su base clinico-diagnostica dello standard, da proporre al paziente per una collaborazione e per definire il processo tagliato su misura. È uno strumento molto impegnativo non ancora pienamente valutato. Il secondo problema è la lentezza con cui le LG sono aggiornate e con cui vengono traslate nei PDTA e ciò è molto importante nel momento in cui nuove terapie e nuovi Companion Diagnostics vengono continuamente proposti [30]. 


\section{PDTA e Medicina di Laboratorio}

Cinque anni fa organizzazioni diverse concordarono, seppur con differenze concettuali importanti, sui quattro ruoli principali del Laboratorio nella Medicina contemporanea: sacerdote della tecnologia, manageriale, consulente clinico, attore del governo clinico. Il ruolo di attore del governo clinico si esprimeva nella definizione collaborativa di "evidenze", linee guida, EBLM e PDTA, oltre che nella formazione di specialisti e medici di medicina generale (MMG) [31]. Ma, nel concreto, come si traduce il ruolo della Medicina di Laboratorio rispetto ai PDTA? La risposta richiede di tenere presenti due principali aspetti: la predominanza dei PDTA terapeutici o in cui questa parte è preponderante e il diversificato impatto possibile del Laboratorio sulle differenti fasi della costruzione di un PDTA.

Appare piuttosto ovvio che il Laboratorio partecipi alla definizione di un PDTA, data la sua importanza nella Medicina contemporanea. È diventata citazione comune quella che riferisce essere il Laboratorio decisivo per il $60-70 \%$ delle decisioni cliniche. In realtà Forsman [32], che nel 1996 per primo ha indicato tali valori senza peraltro fornire riferimenti bibliografici o di studio se non la quantità di spazio occupato dai dati di laboratorio negli archivi digitali delle cartelle cliniche, si riferisce alle decisioni "critiche" ospedaliere e, quindi, questo valore non può essere trasferito toutcourt a tutte le decisioni cliniche, come normalmente si fa dopo l'uso del dato nel documento della Pathology Modernisation inglese [33] e nella ricerca di The Lewin Group [34] del 2005. Questo errato utilizzo di un dato non evidencebased è stato segnalato da Autori sia italiani [35] sia inglesi [36] e tuttavia permane non solo tra i profani, ma anche nella letteratura scientifica. Recentemente, infatti, Rohr et al [37] accreditano il concetto che il $66 \%$ delle decisioni cliniche sarebbe guidato dagli esami di laboratorio. In effetti, si tratta del 66\% di diagnosi in pazienti di una ristretta coorte di oncologi e cardiologi americani e tedeschi (n. 79), quindi di un dato non generalizzabile. Infatti, uno studio tedesco [38], citato anche da Rohr et al [37], ha dimostrato che il 32\% (187 su 584) delle diagnosi cliniche esaminate viene confermato dal solo uso di esami di laboratorio. E se ci si muove in ambito territoriale, $i$ dati al di qua e al di là dell'Atlantico $[39,40]$ concordano nell' indicare tra il $20 \%$ e il $30 \%$ la percentuale di pazienti ambulatoriali per i quali il MMG prescrive esami di laboratorio. In conclusione, l'impatto della Medicina di Laboratorio è molto variabile secondo il tipo di paziente e del suo stato, dei setting in cui viene visto e dei medici/team assistenziale che lo prende in carico. Di conseguenza, anche nei PDTA l'impatto del Laboratorio, in generale, è molto variabile. Tuttavia, esistono PDTA cardiologici, renali, oncologici, ematologici e infettivologici in cui l'esame di laboratorio è il pivot del percorso del paziente. Un'analisi dei 454 CP raccolti da NICE [23] mostra che almeno il 16\% ha nella Medicina di Laboratorio il cardine del percorso.

Il coinvolgimento del Laboratorio nel gruppo di lavoro multidisciplinare è, peraltro, opportuno anche quando gli esami di laboratorio non sono il pivot del PDTA, in particolare in riferimento alla ricerca e valutazione delle LG da tradurre in protocolli. Infatti, è noto [41] come le LG cliniche abbiano generalmente una bassa considerazione per gli aspetti considerati laboratoristici, rispettando in media solo in un terzo dei casi le necessarie informazioni riguardo lo stato dei pazienti, le interferenze biologiche e analitiche e il trattamento dei campioni, e che l'inserimento di uno specialista di laboratorio nei comitati/gruppi di preparazione di LG cliniche migliora la situazione rendendone circa la metà esaustiva sotto il profilo dei mezzi diagnostici. È quindi spesso necessario "adattare" LG cliniche, utilizzando in maniera sistematica e ragionata quanto disponibile in letteratura per fornire protocolli utili ai professionisti della Medicina di Laboratorio a risolvere i concreti problemi giornalieri riguardo i PDTA e ai clinici per una interpretazione corretta del significato dell'esame. Da questo punto di vista, l'importanza dell' accuratezza diagnostica è stata recentemente ribadita $[42,43]$ anche e soprattutto in riferimento ai PDTA, nella sua interrelazione con la performance clinica, l'effectiveness clinica, la costo-efficacia e con l'impatto "sociale" del test, nel definire le reciproche e dinamiche influenze con i PDTA. Gli esempi riportati vanno dall'utilizzo di esami reflex all'uso di iPTH, di hs-cTn versus cTn in diagnosi e prognosi, di diversi limiti decisionali di $\mathrm{Hb}_{\mathrm{A} 1}$ per diagnosi o monitoraggio, di CRP in sostituzione di VES, di PNC in scompenso acuto e cronico di cuore e di POCT per $\mathrm{Hb}_{\mathrm{Alc}}$, ACR, PTT/INR, HIV e TBC ecc. Le difficoltà di collegare direttamente le caratteristiche dei test all'outcome sono state ben illustrate, come d'altra parte le strategie che si possono utilizzare nella valutazione dei test $[42,43]$.

L'applicazione alla realtà locale di protocolli derivati da LG internazionali è modulata dalla peculiarità della situazione italiana/aziendale e dalla necessità di tagliare su misura del target del PDTA le LG selezionate, ma necessita poi di manutenzione alla luce dei tempi di invecchiamento delle LG che hanno una mediana di obsolescenza pari a 5,5 anni, ma i primi segni di cedimento dopo 2 anni per almeno un quarto di esse [44]. Inoltre, l'innovazione tecnologica è spesso più veloce per il Laboratorio che per la realtà clinica e, ancor più, per gli enti istituzionali. Vi è dunque la necessità di una continua attenzione del Laboratorio per l'introduzione, evidence based, di nuovi marcatori all'interno di PDTA già in uso per rispondere a bisogni clinici fino a quel momento non soddisfatti. È il tema emergente degli "unmet clinical needs" definiti come "any missing or inadequately performing component of a clinical pathway". Il metodo per l'individuazione e valutazione di biomarcatori in grado di rispondere a bisogni clinici insoddisfatti è stato ampiamente trattato [45], con esempi riferiti al test rapido per 
Chlamydia trachomatis in Medicina generale per la prevenzione della malattia infiammatoria pelvica e alla determinazione della fibronectina fetale nella gestione della minaccia di parto pretermine. Questi aspetti sono particolarmente importanti alla luce dell'avanzata della Medicina di Laboratorio " $4 P s$ " con i nuovi test e/o i nuovi livelli diagnostici della Medicina di Laboratorio personalizzata e/o di precisione e al rimarcato principale problema di tanti PDTA (la mancata innovazione).

Infine, l'apporto della Medicina di Laboratorio ai PDTA è cruciale rispetto al tema della centralità del paziente e ai suoi determinanti per la qualità del servizio, quali l'accessibilità in termini di fase preanalitica (preparazione ai test; prelievo; ambulatori specialistici; selezione dei test), la risposta al quesito clinico come organizzazione e comunicazione dei risultati (compresi i percorsi interni proattivi del Laboratorio e i limiti decisionali nell'algoritmo del PDTA), l'informazione/formazione all'interpretazione del team assistenziale (non tutto necessariamente dotato di conoscenze specifiche sufficienti) e la consulenza diretta al paziente [2].

In conclusione, la Medicina di Laboratorio deve fare la sua parte nella costruzione dei PDTA, partecipando ai gruppi multidisciplinari e multiprofessionali, con attenzione ai test pivot, al loro uso e alla loro interpretazione; alla qualità delle LG scelte; al dettaglio dei protocolli operativi che sostanziano il percorso; agli aspetti "interni" che determinano (performance analitica) o aumentano (total testing process) la qualità della risposta; alla verifica continua dell'appropriatezza e adeguatezza dei marcatori utilizzati e all'audit dell'effectiveness del PDTA. Si tratta di uno degli aspetti più alti di "lavoro all'interfaccia", nelle aree di pre-pre e postpost testing process, ove l'affidabilità analitica e il suo rapporto con la realtà clinica e i suoi esiti è immediatamente percepibile.

\section{Conflitti di interesse Nessuno.}

Studi condotti su esseri umani e animali Per questo tipo di studio non è richiesto l'inserimento di alcuna dichiarazione relativa agli studi effettuati su esseri umani e animali.

\section{Bibliografia}

1. Gerteis M, Edgman-Levitan S, Daley J et al (1993) Through the patient's eyes: understanding and promoting patient-centered care. Jossey-Bass, San Francisco

2. Cappelletti P (2016) Medicina di Laboratorio centrata sul paziente. Riv Ital Med Lab 12:193-197

3. Wagner EH, Austin BT, Von Korff M (1996) Organizing care for patients with chronic illness. Milbank Q 74:511-544

4. Wagner EH, Davis C, Schaefer J et al (1999) A survey of leading chronic disease management programs: are they consistent with literature? Manag Care Q 7:56-66

5. WHO (2002) Innovative care for chronic conditions: building blocks for action. Geneva
6. Coleman K, Austin BT, Brach C et al (2009) Evidence on the Chronic Care Model in the new millennium. Health Aff (Millwood) 28:75-85

7. Campbell H, Hotchkiss R, Bradshaw $N$ et al (1998) Integrated care pathways. BMJ 316:133-137

8. Singer SJ, Burgers J, Friedberg M et al (2011) Defining and measuring integrated patient care: promoting the next frontier in health care delivery. Med Care Res Rev 68:112-127

9. http://e-p-a.org/sito-internet-e-p-a/percorsi-diagnosticiterapeutici-e-assistenziali-pdta/ (Accesso 21 maggio 2017)

10. De Luc K (2001) Developing care pathways - the handbook. Radcliffe Medical Press, Oxford

11. De Bleser L, Depreitere R, De Waele K et al (2006) Defining pathways. J Nurs Manag 14:553-563

12. Vanhaecht K, De Witte K, Depreitere R et al (2006) Clinical pathway audit tool: a systematic review. J Nurs Manag 14:529-537

13. Kinsman L, Rotter T, James E et al (2010) What is a clinical pathway? Development of a definition to inform the debate. BMC Med 8:31-33

14. http://www.evidence.it/articolodettaglio/210/it/44/linee-guidapercorsi-processi-procedure-protocolli-il-caos-/articolo (Accesso 21 maggio 2017)

15. Chawla A, Westrich K, Matter S et al (2016) Care Pathways in US Healthcare Settings: current successes and limitations, and future challenges. Am J Manag Care 22:53-62

16. Gagliardi AR, Brouwers MC, Palda VA et al (2011) How can we improve guidelines use? A conceptual framework of implementability. Implement Sci 6:26-37

17. Dong W, Huang Z (2015) A method to evaluate critical factors for successful implementation of Clinical Pathways. Appl Clin Inform 6:650-668

18. Bjurljng-Sjöberg P, Wadensten B, Pöder U et al (2015) Factors affecting the implementation process of clinical pathways: a mixed method study within the context of Swedish intensive care. J Eval Clin Pract 21:255-261

19. Sather EW, Svindseth MF, Sorthe I et al (2016) Barriers and success factors in clinical pathways: transition process between psychiatric inpatient care and community care. European Journal for Person Centered Healthcar 4:631-640

20. Böckmann B, Heiden K (2013) Extracting and transforming clinical guidelines into pathway models for different hospital information systems. Health Inf Sci Syst 1:13-21

21. Ellis PG (2013) Development and implementation of oncology care pathways in an integrated care network: the VIA oncology pathway experience. J Oncol Pract 9:171-173

22. Richter P, Burwitz M, Esswein W (2016) Conceptual considerations on the integration of quality indicators into clinical pathways. Stud Health Technol Inform 228:38-42

23. https://www.nice.org.uk/About/What-we-do/Our-Programmes/ About-NICE-Pathways (Accesso 21 maggio 2017)

24. Paparella M (2014) Il supporto dell'ICT ai PDTA: una prima analisi empirica. https://www.fiaso.it/News/Ricerca-studi-eindagini/Con-1-ICT-nei-percorsi-assistenziali-piu-aderenza-alleterapie-e-minori-costi (Accesso 21 maggio 2017)

25. Vanhaecht V (2007) The impact of Clinical Pathways on the organisation of care processes. https://lirias.kuleuven.be/bitstream/ 123456789/252816/1/PhD+Kris+Vanhaecht.pdf (Accesso 21 maggio 2017)

26. Mater W, Ibrahim R (2014) Delivering quality healthcare services using clinical pathways. International Journal of Computer Applications 95:5-8

27. Rotter T, Kinsman L, James E et al (2012) The effects of Clinical Pathways of professional practice, patient outcomes, length of stay, and hospital costs: cochrane systematic review and meta-analysis. Evaluation and the Health Professions 35:3-27 
28. Schnipper LE, Davidson NE, Wollins DS et al (2015) American society of clinical oncology statement: a conceptual framework to assess the value of cancer treatment options. J Clin Oncol 23:2563-2577

29. Dubuc N, Bonin L, Tourigny A (2013) Development of integrated care pathways: toward a care management system to meet the needs of frail and disabled community-dwelling older people. Int J Integr Care 13:e017

30. Wurcel V, Perche O, Lesteven D et al (2016) The value of Companion Diagnostics: overcoming access barriers to transform personalized health care into an affordable reality in Europe. Public Health Genomics 19:137-143

31. Cappelletti P (2012) Scenari e ruoli della Medicina di Laboratorio ai tempi della crisi: da Modena a Salerno ed oltre. Riv Ital Med Lab 8:183-189

32. Forsman RW (1996) Why is the laboratory an afterthought for managed care organizations? Clin Chem 42:813-816

33. Report of the Review of NHS Pathology Services in England (2006) An Independent Review for the Department of Health. Department of Health, London

34. The Lewin Group (2005) The value of diagnostics. AdvaMed, Washington

35. Cappelletti P (2007) La Medicina di Laboratorio e il miglioramento delle cure: un servizio essenziale, una prospettiva interdisciplinare, una visione olistica. RIMeL/IJLaM 3(Suppl):19-24

36. Hallworth MJ (2011) The "70\% claim": what is the evidence base? Ann Clin Biochem 48:487-488
37. Rohr UP, Binder C, Dieterle T et al (2016) The value of in vitro diagnostic testing in medical practice: a status report. PLoS ONE 11:e0149856

38. Wilke MH, Schenker M, Hoffmann G (2002) Detection and documentation of DRG-relevant comorbidities using laboratory tests. Aust Health Rev 25:152-160

39. Cappelletti P (2014) Perché i medici chiedono gli esami di laboratorio? (Poster) $28^{\circ}$ Congresso Nazionale SIMeL. La complessità e la Medicina di Laboratorio. Rimini 29 e 30 ottobre

40. Hickner J, Thompson PJ, Wilkinson T et al (2014) Primary care physicians' challenges in ordering clinical laboratory tests and interpreting results. J Am Board Fam Med 27:268-274

41. Aakre KM, Langlois MR, Watine J et al (2013) Critical review of laboratory investigations in clinical practice guidelines: proposals for the description of investigation. Clin Chem Lab Med 51:12171226

42. Horvath AR, Lord SJ, St John A et al (2014) From biomarkers to medical tests: the changing landscape of test evaluation. Clin Chim Acta 427:49-57

43. Horvath AR, Bossuyt PM, Sandberg S et al (2015) Setting analytical performance specifications based on outcome studies-is it possible? Clin Chem Lab Med 53:841-848

44. Shojania KG, Sampson M, Ansari MT et al (2007) How quickly do systematic reviews go out of date? A survival analysis. Ann Intern Med 147:224-233

45. Monaghan PJ, Lord SJ, St John A et al (2016) Biomarker development targeting unmet clinical needs. Clin Chim Acta 460:211-219 